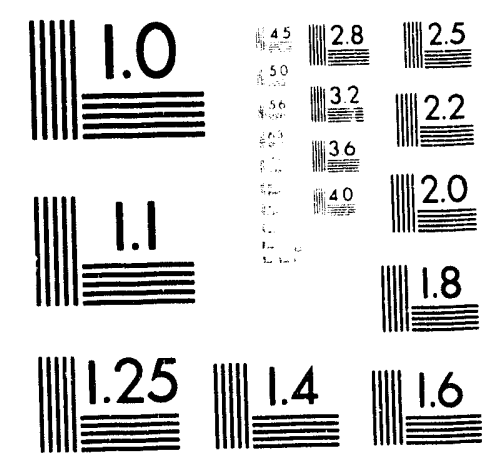



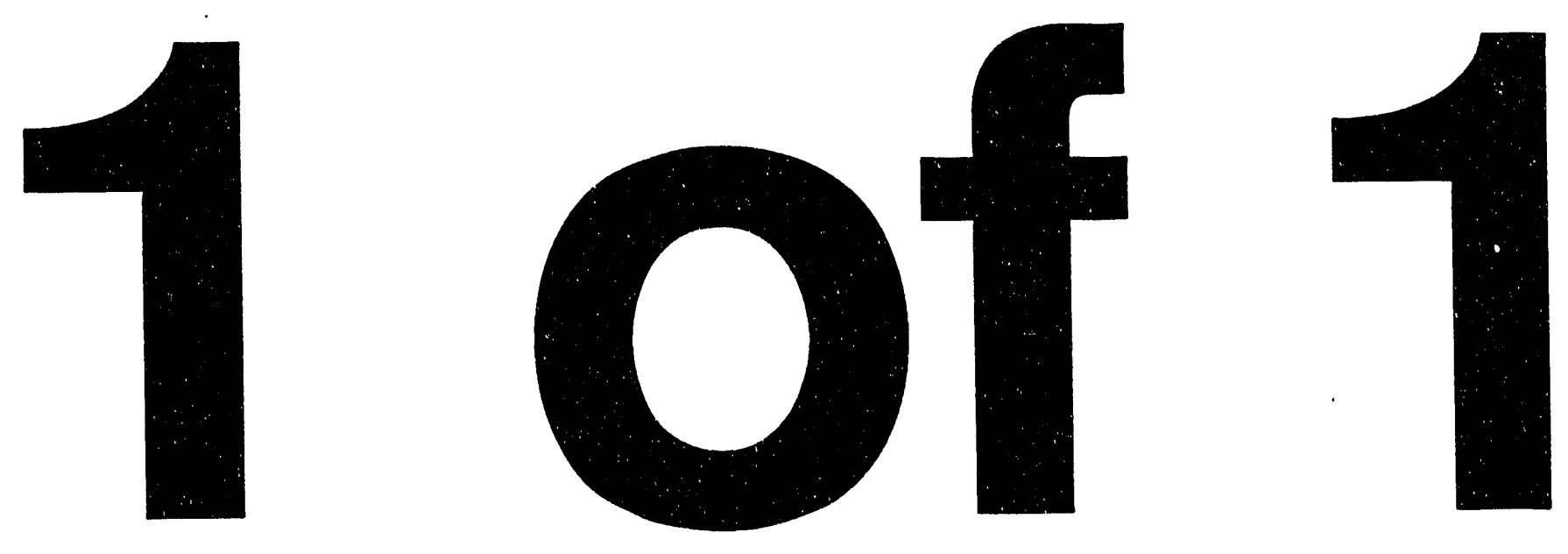
LBL-34107

UC-419

HIFAN-576

\title{
Emittance Growth Rates For Displaced Beams
}

\author{
O. A. Anderson \\ Accelerator \& Fusion Research Division \\ Lawrence Berkeley Laboratory \\ University of California \\ Berkeley, California 94720
}

Submitted to the International Symposium on Heavy

Ion Inertial Fusion, Frascati, Italy, May 25-28, 1993

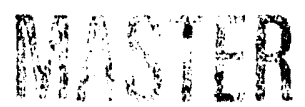

*This work was supported by the Office of Energy Research, Office of Fusion Energy, U.S. Department of Energy under Contract No. DE-AC03-76SF00)98. 


\section{EMITTANCE GROWTH RATES FOR DISPLACED BEAMS \\ O. A. ANDERSON ${ }^{\text {a) }}$ \\ Lawrence Berkeley Laboratory, Berkeley CA 94720 USA}

Emittance growth rates have heen previously analyzed for nonuniform beams in linear channels [1] and for initially uniform mismatched beams in nonlinear channels $[2,3]$. These studies were for centered beams. Additional emittance growth can arise in cases where the beam is initially displaced [4]. The purpose of this study is to obtain growth rates for displaced beams.

This work differs from studies involving random displacement of electrodes [5]. Our analysis assumes instead that the focusing system is perfectly aligned but that the beam is initially displaced with respect to the equilibrium axis. If the focusing force is slightly nonlinear, we find a gradual transfer of the potential energy of beam displacement into kinetic energy associated with emittance growth.

We present explicit results for the emittance growth distance as a function of the nonlinearity of the channel. These results will have practical importance for designers of accelerators and transport systems when setting realistic tolerances for initial beam alignment. These tolerances will depend on the nonlinearity and the length of the system.

\section{INTRODUCTION}

Emittance growth in charged beams is caused by conversion of electrostatic energy into quasi-disordered transverse kinetic energy. The available electrostatic energy has two sources: the free self-field energy, and the free interaction energy between the beam particles and the focusing field. As previously shown by simulations [6] and analytically [1], the self-field energy is released rapidly in one-quarter of a plasma period. The present report treats the much slower process in which interaction energy from beam displacement is converted into rms emittance growth.

We generalize our previous analysis [1] to include beam displacement and nonlinear focusing. Section II gives the solution of the particle equation of motion. We show in section III that the center-of-mass oscillations are damped and derive the damping rate for conversion of potential energy of displacement into internal motion of the beam, i.e., into rms emittance growth.

For simplicity, we analyze the case of uniform sheet beams. Our conclusions (section IV) should also apply to moderately warm round beams; see discussion in Ref. [1]. As before $[1,2]$ we simplify our equations by treating a beam of nonrelativistic particles, all with mass $m$, charge e, and longitudinal velocity $\mathrm{v}$; we assume transverse velocities $\ll v$.

\section{MOTION OF PARTICLES IN A DISPLACED OR MISMATCHED BEAM}

If the initial sheet beam is asymmetric, displaced, and/or mismatched, there will generally be center-of mass oscillations and/or rms beamwidth oscillations. A small nonlinearity in the external field will slowly transfer the energy of these oscillations into emittance growth. An ion has the equation of motion in the presence of a cubic nonlinearity with parameter $v$ :

$$
\mathrm{x}^{\prime \prime}=-\mathrm{k}_{0} 2 \mathrm{x}\left(1+\mathrm{v} \frac{\mathrm{x}^{2}}{\mathrm{q}^{2}}\right)+\frac{\mathrm{P}}{\mathrm{N}} \mathrm{N}_{\mathrm{x}}(\mathrm{x}, \mathrm{z})
$$

where $x^{\prime \prime}=d^{2} x / d z^{2}, P=4 \pi N e^{2} / m v^{2}, k_{0}^{2}$ represents the external focusing force, $q \equiv P / k_{0}{ }^{2}$,

$$
N_{X}(x, z)=\int_{-\infty}^{x} n\left(x_{1}, z\right) d x_{1}-N,
$$

\footnotetext{
a) Also affiliated with Particle Beam Consultants, 2910 Benvenue Ave., Berkeley CA 94705, USA.
} 
and

$$
N=\frac{1}{2} \int_{-\infty}^{\infty} n(x) d x .
$$

The beam is assumed to be thin, i.e., $\mathrm{k}_{0} \mathrm{~h} \ll 1$, and far from conducting plates. We will show that emittance grows on a distance scale $\sim 1 / v$, if the nonlinearity parameter $v$ is small.

Equations (1-3) apply to the general case of asymmetric warm beams; derivation and discussion will be published elsewhere. For simple analytic results, we specialize here to cold laminar beams. We will derive a condition for laminar motion to persist over the long emittancegrowth distance scale so that the last term in Eq. (1) remains constant for each particle. We use the notation $x(0)=\xi$ for initial position and $h$ for the initial beam half-width. The beam is assumed to have initial displacement $\delta \mathrm{x}$ :

$$
-\mathrm{h}+\delta \mathrm{x} \leq \xi \leq \mathrm{h}+\delta \mathrm{x}
$$

With the notation $\delta \equiv \delta x / h$, and the beam density $n(x)$ assumed initially uniform,

$$
\frac{N_{X}}{N}=\frac{\xi}{h}-\delta \equiv \chi
$$

with $-1 \leq \chi \leq 1$. 'The last term in Eq. (1), which becomes $P \chi$, can be eliminated by a change of coordinate,

which gives

$$
y=\frac{x-x_{e}}{q}
$$

if

$$
\frac{\mathrm{y}^{\prime \prime}}{\mathrm{k}_{0}^{2}}+\left(1+3 v \frac{\mathrm{x}_{\mathrm{e}}^{2}}{\mathrm{q}^{2}}\right) \mathrm{y}+3 v \frac{\mathrm{x}_{\mathrm{e}}}{\mathrm{q}} \mathrm{y}^{2}+v \mathrm{y}^{3}=0
$$

$$
\frac{x_{e}}{q}=\chi-v\left(\frac{x_{e}}{q}\right)^{3}=\chi-v \chi^{3}+O\left(v^{2}\right) .
$$

Eq. (7) can be solved to high accuracy by the Lindstedt-Poincare method if the initial beam displacement and mismatch are small. We define the small parameter, related to mismatch,

To first order in $\delta, \varepsilon$, and $\nu$, the solution is

$$
\varepsilon=\frac{\mathrm{h}}{\mathrm{q}}-1 \text {. }
$$

$$
\mathrm{x}(\chi, L)=\mathrm{q} \chi\left(1-v \chi^{2}\right)+\mathrm{q}\left(\delta+\varepsilon \chi+v \chi^{3}\right) \cos \left[\left(1+\frac{3}{2} v \chi^{2}\right) \mathrm{k}_{0} \mathrm{z}\right] .
$$

Phase Space Configurations for Displaced or Mismatched Beams.

Differentiating Eq. (10) with respect to $z$ gives, to lowest order,

$$
x^{\prime}(\chi, z)=-k_{0} q\left(\delta+\varepsilon \chi+v \chi^{3}\right)\left(1+\frac{3}{2} v \chi^{2}\right) \sin \left[\left(1+\frac{3}{2} v \chi^{2}\right) k_{0} z\right]
$$

which, together with Eq. (10), gives the $\left(x, x^{\prime}\right)$ phase space configuration for any $z<z_{\text {crit }}$. Particles in different regions oscillate at different frequencies and become increasingly out of step. The phase-space configuration becomes increasingly irregular, causing rms emittance growth. Figure 1 shows the degree of development of emittance irregularity after 16 plasma periods (i.e., $\mathrm{k}_{0} z / 2 \pi=16$ ) for the displaced beam case. 
Laminarity. Laminar motion implies

$$
\mathrm{n}(\mathrm{x}, \mathrm{z}) \mathrm{dx}=\mathrm{n}(\xi) \mathrm{d} \xi .
$$

The distance between two particles relative to their small initial separation is $\partial \mathrm{x} / \partial \xi$; trajectories cross at the point where this ratio vanishes. From Eq. (10),

$$
\frac{\partial \mathrm{x}}{\partial \xi}=\frac{\mathrm{q}}{\mathrm{h}}\left\{1-3 v \chi^{2}+\left(\varepsilon+3 v \chi^{2}\right) \operatorname{coskz}-3 v \mathrm{k}_{0} z \chi\left(\delta+\varepsilon \chi+v \chi^{3}\right) \sin \left[\left(1+\frac{3}{2} v \chi^{2}\right) \mathrm{k}_{0} z\right]\right\} .
$$

The reciprocal of Eq. (13) gives the beam density, which becomes singular when trajectory crossing first occurs. A study of Eq. (13) shows that this first happens at the beam edge where $\chi=1$ and at the point where $\cos \left[(1+3 v / 2) \mathrm{k}_{0} \mathrm{z}\right] \approx 0$. Then $\partial \mathrm{x} / \partial \xi=0$ gives the critical distance

$$
\mathrm{k}_{0} \mathrm{z}_{\text {crit }}=\frac{1}{3 v} \frac{1-3 v}{\delta+\varepsilon+v} \text {. }
$$

We will see in section III that most of the emittance growth usually occurs for $\mathrm{z}<\mathrm{z}_{\text {crit }}$.

\section{DAMPING RATE OF CENTER-OF-MASS MOTION FOR DISPLACED BEAM}

Equation (10) gives the motion of each beam particle. We get the center-of-mass motion by averaging over all the particles. Averages over density profiles are defined by

$$
\langle\mathrm{g}\rangle(\mathrm{z})=\frac{1}{2 \mathrm{~N}} \int_{-\infty}^{\infty} \mathrm{d} \mathrm{x} n(\mathrm{x}, \mathrm{z}) \mathrm{g}(\mathrm{x}) .
$$

During the period of laminar flow, we can use Eq. (12) to change the variable of integration from $x$ to the initial value $\xi$ :

$$
\langle\mathrm{g}\rangle(\mathrm{z})=\frac{1}{2 \mathrm{~N}} \int_{-\infty}^{\infty} \mathrm{d} \xi \mathrm{n}(\xi) \mathrm{g}(\mathrm{x}(\xi, \mathrm{z}))=\frac{1}{2} \int_{-1}^{1} \mathrm{~d} \chi \mathrm{g}(\mathrm{x}(\chi, \mathrm{z})),
$$

where in the last expression we changed the integration variable from $\xi$ to $\chi$ and recalled that the initial density was assumed uniform for $-1 \leq \chi \leq 1$. Applying Eq. (16) to Eq. (10), we note that the four odd terms drop out by symmetry. The remaining even term can be integrated from 0 to 1 . The sum-of-angles rule gives

where

$$
\frac{\langle x\rangle}{\mathrm{q}}=\delta \int_{0}^{1} \mathrm{~d} \chi\left[\cos \mathrm{k}_{0} \mathrm{z} \cos \frac{\pi}{2} \psi^{2} \chi^{2}-\sin \mathrm{k}_{0} \mathrm{z} \sin \frac{\pi}{2} \psi^{2} \chi^{2}\right]
$$

$$
\psi=\sqrt{(3 / \pi) \mathrm{vk}_{0} \mathrm{z}} .
$$

Changing the integration variable once more gives the standard forms for Fresnel integrals

$$
\frac{\langle\mathrm{x}\rangle}{\mathrm{q}}=\frac{\delta}{\psi} \int_{0}^{\psi} \mathrm{dt}\left[\cos \frac{\pi}{2} \mathrm{t}^{2} \cos \mathrm{k}_{0} \mathrm{z}-\sin \frac{\pi}{2} \mathrm{t}^{2} \sin \mathrm{k}_{0} \mathrm{z}\right] \equiv \frac{\delta}{\psi}\left[\mathrm{C}(\psi) \cos \mathrm{k}_{0} \mathrm{z}-\mathrm{S}(\psi) \sin \mathrm{k}_{0} \mathrm{z}\right] .
$$

The Fresnel integrals $C(\psi)$ and $S(\psi)$ are discussed in Abramowitz and Stegun [7]. Equation (19) gives damping of center-of-mass oscillations due to the increasing denominator $\psi$, as illustrated in Fig. 2. The oscillation energy is transferred into emittance growth at a rate determined by $v$.

For large $\psi, C(\psi)$ and $S(\psi) \rightarrow 0.5$ and Eq. (19) becomes 


$$
\frac{1}{\mathrm{q}} \mathrm{x}_{\mathrm{av}}(\mathrm{z})=\frac{\langle\mathrm{x}\rangle}{\mathrm{q}} \rightarrow 0.5 \frac{\delta}{\psi}\left(\cos \mathrm{k}_{0} \mathrm{z}-\sin \mathrm{k}_{0} \mathrm{z}\right)
$$

or

$$
\frac{x_{a v}}{q \delta} \rightarrow \frac{\cos \left(k_{0} z-\pi / 4\right)}{\sqrt{(6 / \pi) v k_{0} z}}=\frac{\cos \left(k_{0} z-\pi / 4\right)}{\sqrt{12 v \alpha}}
$$

where we define the normalized length $\alpha$, equal to the number of cold-beam plasma periods

$$
\alpha \equiv \frac{\mathrm{k}_{0} \mathrm{z}}{2 \pi} \text {. }
$$

The ratio of the interaction energy to its initial value is $x_{a v}{ }^{2} / \delta^{2}$; this ratio takes on a small value at $\mathrm{z}_{\text {crit }}$, according to Eqs. (14) and (22). Therefore the energy is mostly transferred to emittance growth while the motion is laminar.

Damping length (emittance growth length) for center-of-mass oscillations:

Corresponding to $1 / \sqrt{12 v \alpha}=1 / \mathrm{e}$, we have the final result in terms of plasma periods

$$
\alpha_{\text {growth }} \cong \frac{0.6}{v} \text {. }
$$

Simulations of emittance vs. distance [8] show saturation on the distance scale predicted by Eq. (23). The maximum emittance that can be attained is easily found by energy conservation, as was done previously for centered beams [2].

\section{CONCLUSIONS}

Our result, Eq. (23), can be written in terms of the number of focusing cells traversed by the beam. For a cold beam in a quadrupole channel, one has approximately $\alpha \sim \sigma_{0} \mathrm{z} / 2 \pi \ell$, where $\sigma_{0}$ is the vacuum phase advance per cell length $\ell$. Then the number of cells is $n=z / l=2 \pi \alpha / \sigma_{0}$, so that if the undepressed tune is $57^{\circ}$, i.e., if $\sigma_{0}=1$,

$$
\mathrm{n}_{\text {growth }}=2 \pi \alpha_{\text {growth }}=\frac{1.2 \pi}{v} \text {. }
$$

We have used Eq. (23) and assumed that it is reasonably accurate for round beams. For $v=0.05$, the emittance growth occurs over about 75 cells. If the beam energy changes along the channel, one would use some sort of averaging to estimate the growth length.

Warm beams: As discussed in Ref. [8], fluid motion is essentially unaffected by beam temperature if the tune depression $\sigma / \sigma_{0}<1 / \pi \sqrt{3}$. In the present case, a good criterion is $\epsilon_{0}<0.5 \in_{\mathrm{eg}}$, where $\epsilon_{0}$ and $\epsilon_{\mathrm{eg}}$ are the initial and final emittances.

Round heams: The physics of emittance growth has been found to be quite similar for round beams and sheet beams [1], although, of course, sheet beams are mathematically simpler. The source of emittance growth treated in this paper (free interaction energy) applies to both types of beams, and we expect the same damping mechanism to prevail. The damping length calculated in this paper should provide reasonable estimates for either sheet beams or round beams.

\section{ACKNOWLEDGMENTS}

I wish to thank W. S. Cooper and E. P. Lee for their support and their comments on this paper. This work was supported in part by U.S. DOE Contract DE-AC03-76SF00098. 


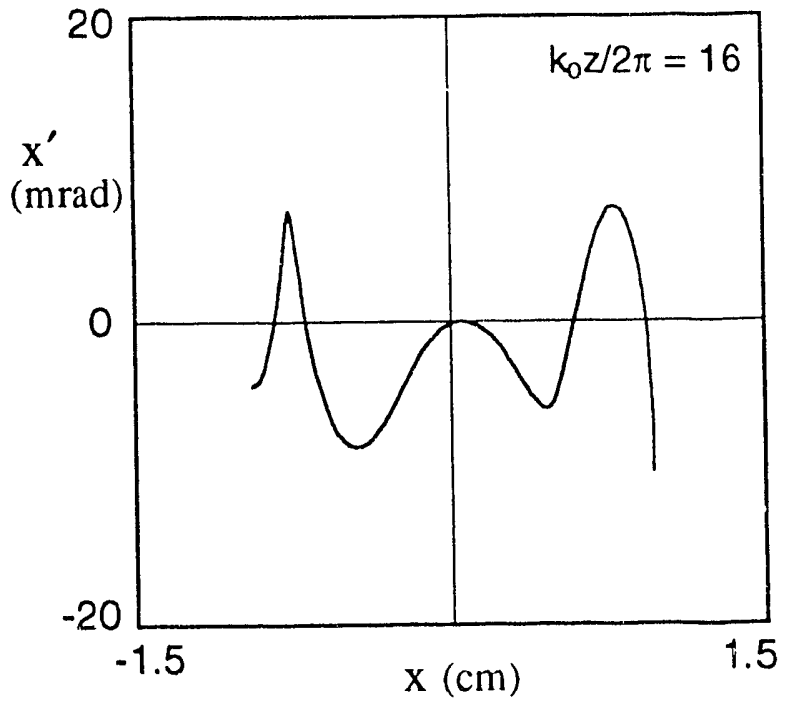

Fig. 1. Emittance diagram plotted from Eqs. (10) and (11) after 16 plasma periods. Nonlinearity coefficient $v=0.05$; displacement factor $\delta=0.05$; mismatch factor (Ref. [8]) $\mu=\varepsilon+3 v / 5=0$.

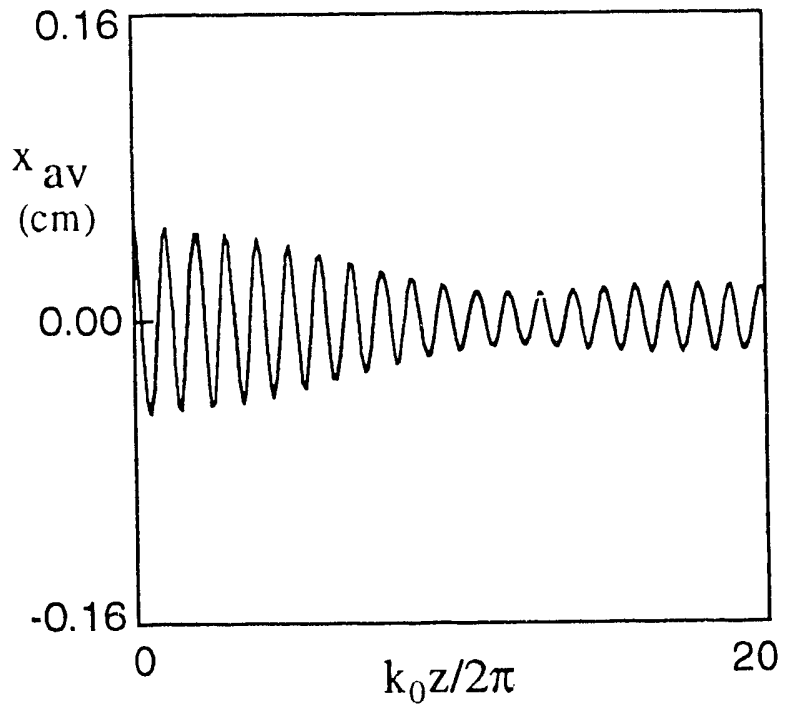

Fig. 2. Damping of center-of-mass oscillations, from Eq. (19). Nonlinearity $v=0.05$; displacement factor $\delta=0.05$; mismatch factor (Ref. [8]) $\mu=\varepsilon+3 v / 5=0$.

\section{REFERENCES}

[1] O.A. Anderson, Particle Accelerators 21, 197 (1987).

[2] O.A. Anderson, Proc. International Symp. on Heavy Ion Fusion, M. Reiser, Editor. (AIP Conf. Proc. 152, p. 253, 1986).

[3] O.A. Anderson and L. Soroka, Proc. 1987 Particle Accelerator Conf., (IEEE Cat. No. 87CH2387-9, p. 1043, 1987).

[4] Martin Reiser, J. Appl. Phys. 70, 1919 (1991).

[5] Lloyd Smith, Lawrence Berkeley Laboratory internal reports LBID-940 (1984) and HIFAR Note-192 (1988); also E.P. Lee, personal communication, LBL (1992).

[6] T.P. Wangler, K.R. Crandall, R.S. Mills, and M. Reiser, IEEE Trans. Nucl. Sci. 32, 2196 (1985).

[7] M. Abramowitz and I.A. Stegun, Handbook of Mathematical Functions, Dover Publications, New York, 1970.

[8] O.A. Anderson, Lawrence Berkeley Laboratory report LBL-34082, to be submitted to Particle Accelerators . 

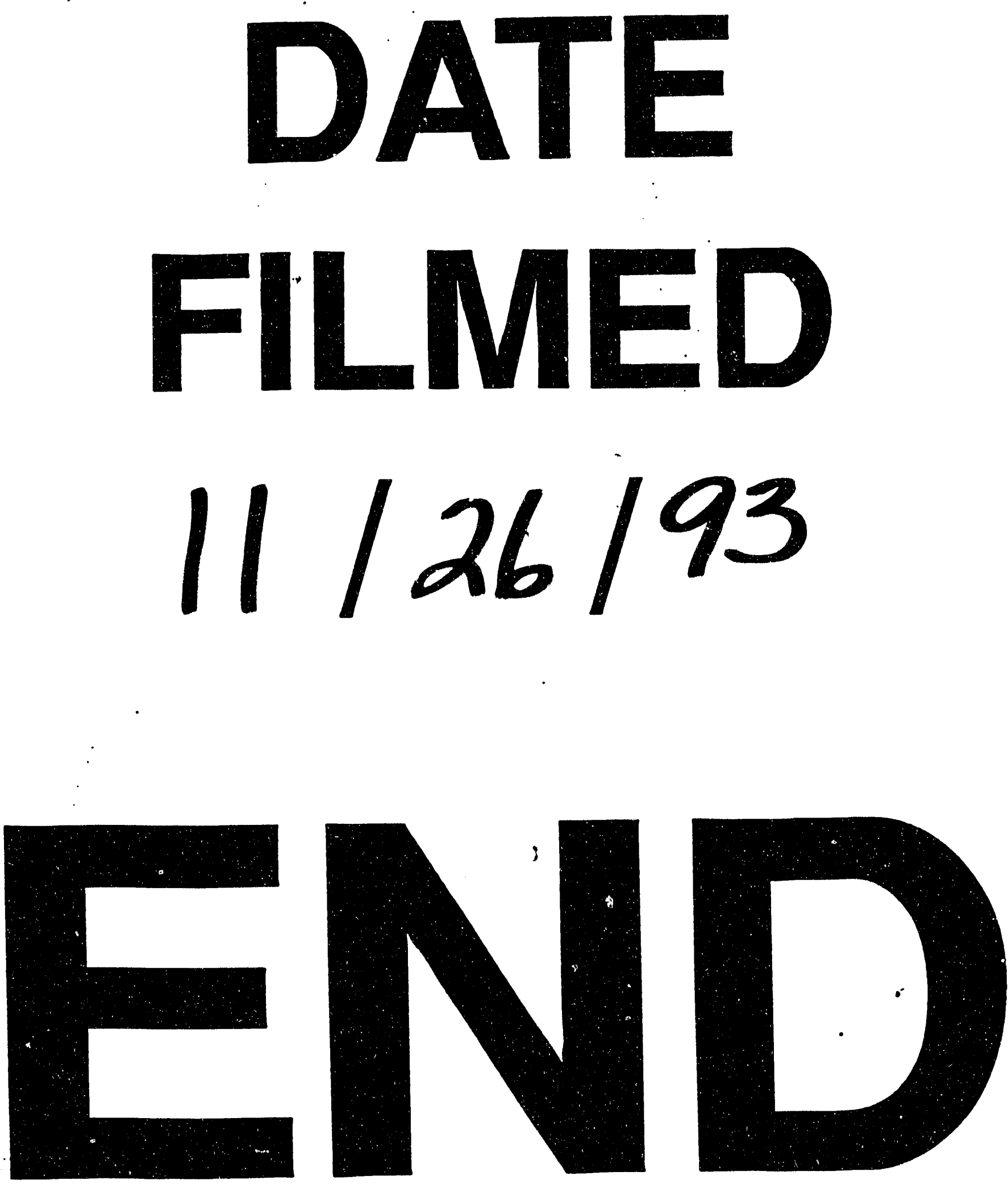
